\title{
BMJ Open Participatory health through behavioural engagement and disruptive digital technology for postoperative rehabilitation: protocol of the PATHway trial
}

\author{
Xia Wang (D) , ${ }^{1}$ David J Hunter (D) , ${ }^{2}$ Sarah Robbins, ${ }^{1}$ Sarah Capistrano, ${ }^{1}$ \\ Vicky Duong, ${ }^{1}$ Luciano Melo, ${ }^{3}$ Anthony Harris, ${ }^{4}$ Manuela Ferreira ${ }^{5}$
}

To cite: Wang $X$,

Hunter DJ, Robbins S, et al. Participatory health through behavioural engagement and disruptive digital technology for postoperative rehabilitation: protocol of the PATHway trial. BMJ Open 2021;11:e041328. doi:10.1136/ bmjopen-2020-041328

- Prepublication history and supplemental material for this paper are available online. To view these files, please visit the journal online (http://dx.doi org/10.1136/bmjopen-2020041328).

Received 08 June 2020 Revised 24 November 2020 Accepted 10 December 2020

Check for updates

(C) Author(s) (or their employer(s)) 2021. Re-use permitted under CC BY-NC. No commercial re-use. See rights and permissions. Published by BMJ.

For numbered affiliations see end of article.

Correspondence to

Dr Xia Wang;

xia.wang@sydney.edu.au

\section{ABSTRACT}

Introduction Postsurgical rehabilitation is critical for optimal recovery in people undergoing orthopaedic surgery. Currently, knee and lumbar spine postsurgical care is not standardised, economically sustainable, nor based on quality evidence, contributing to substantial clinical variation, poor outcomes and increasing healthcare costs. This protocol describes the design of a randomised controlled trial aiming to evaluate the effectiveness and cost-effectiveness of a postsurgical clinical pathway augmented by disruptive technology and compared with standardised rehabilitation alone, in decreasing pain and improving function after total knee replacement (TKR) or lumbar laminectomy (with or without fusion).

Methods An assessor-blinded, parallel group, randomised controlled trial will be conducted to recruit 204 consenting participants (102 per arm) of whom $50 \%$ are undergoing TKR and $50 \%$ lumbar surgery. The intervention group will receive a 6-month technology-enabled rehabilitation package in addition to usual postsurgical care. The package includes (1) an exercise program delivered via the Physitrack app on the iPad, (2) a health-coaching program delivered via video calls and motivational messages, (3) use of physical activity tracker with goal setting and motivational reminders (Fitbit). For those undergoing TKR, the intervention will also include knee joint range of motion self-monitoring via the Goniometer app. The control group will receive usual postsurgical care. Participants will be followed up at 3, 6 and 12 months from the enrolment date. The primary outcome is pain measured with the Numerical Rating Scale at 3 months. Secondary outcomes include pain-related disability, quality of life, computer self-efficacy, physical activity participation and sedentary behaviour. Data analysis will be blinded and by intention-to-treat. A trial-based cost-effectiveness analysis will determine the potential incremental cost per qualityadjusted life-year gained.

Ethics and dissemination This protocol is approved by the human research ethics committee of the University of Sydney. Dissemination will occur through lay summary, infographics, conferences and journal publications.

Trial registration number ACTRN12618001448235.

\section{Strengths and limitations of this study}

- The use of a standardised clinical pathway added to a package of affordable technologies has the potential of improving postsurgical rehabilitation with direct impact on the current healthcare burden.

- This study is adequately powered to provide highquality evidence of cost-effective care after knee and spinal surgeries.

- This study uses remote monitoring devices and structured multiple-stage goals to make sure the intervention is tailored and also standardised for implementation in practice.

- This study is limited to participants who have internet access and are familiar with smart devices.

\section{INTRODUCTION}

\section{Background and rationale}

Musculoskeletal (MSK) disorders are the leading cause of disability and currently affect over 1.3 billion people globally. ${ }^{1}$ Every year in Australia, over 550000 elective surgical procedures are performed for all MSK conditions, one in seven being for knee osteoarthritis (OA) or low back pain. ${ }^{2}$ According to the Australian Medicare Statistics report, 39490 total knee replacements (TKR) and 26315 laminectomies were performed in 2019. ${ }^{3}$ This practice accounts for the majority of the $\$ 6$ billion spent every year on healthcare for these conditions. ${ }^{4}$ Reoperation and hospital readmission following elective knee and spinal surgery are also common due to comorbidities or postsurgical complications ${ }^{5}$ which add substantially to the associated financial burden.

With the rising prevalence of these conditions and ensuing surgical costs, the microscope reviewing the quality of care and cost has sharply focused its lens on this problem. 
There is substantial variation in postsurgical care for the knee and spine surgery in Australia. ${ }^{6}$ This is largely due to the lack of scientific evidence supporting practice in this field and contributes to poor outcomes, and increased healthcare costs. ${ }^{7}$ The variations in care range from the indications for inpatient postsurgical rehabilitation, to outpatient postsurgical rehabilitation, the length of inpatient care and types of physical therapies used. ${ }^{7}$ Patients cannot benefit from treatments that are not developed or delivered according to the best available evidence. This level of variation in care often results in a discrepancy of treatment outcomes and increased healthcare costs, not to mention confusion and in turn patient disengagement and lack of adherence to rehabilitative care.

Compliance with rehabilitation physiotherapy after TKR is dependent on motivation or self-management capacity. ${ }^{8}$ Preliminary studies of technology systems that integrate real-time biofeedback following joint replacement lead to measurable gains in mobility ${ }^{9}{ }^{10}$ Wearable devices to monitor physical activity are feasible following joint replacement ${ }^{11}$ and their use with feedback has been shown to increase participation in physical activity by approximately $20 \%$ in persons with knee $\mathrm{OA}^{12}$ and chronic low back pain. ${ }^{13}$ Mobile health apps are being used by an increasing number of primary healthcare professionals and organisations due to their monitoring and treatment delivery capabilities, while also serving as a cost-effective health service delivery. ${ }^{14}$ Currently, there is small to moderate evidence that these apps contribute to the uptake of physical activity by the user. ${ }^{15} 16$ We recently conducted a systematic review on the effectiveness of using disruptive technologies to assist postsurgical rehabilitation and found that innovative technologies, such as telerehabilitation, virtual reality biofeedback and educational software, reduce joint pain and improve physical function for people undergoing TKR. ${ }^{17}$ However, the quality of the included trials is relatively poor, and there is a lack of evidence for postsurgical care after spinal surgery. Moreover, the majority of the trials investigated telehealth only, and it is still unclear if packaging a variety of technologies with automatic monitoring and motivational messaging will lead to greater benefits following a rehabilitation programme. Reducing clinical variation through standardised clinical pathways and the application of technologies to enhance delivery and motivation are opportunities for improving usual care as well as lessening the ever-increasing burden on the healthcare service.

\section{Objective}

The study aims to determine the effect and costeffectiveness of a postsurgical clinical pathway augmented by a disruptive technology package compared with standardised rehabilitation alone in decreasing pain after TKR or lumbar laminectomy (with or without fusion). The intervention will be composed of (1) an exercise programme delivered via health and fitness app, (2) use of wearable physical activity tracker with motivational reminders, (3) a health-coaching programme delivered via a videoconferencing app and motivational messages and (4) knee joint range of motion (ROM) measuring app (only for TKR participants). The control group will be provided with the postsurgical clinical pathway alone.

\section{METHODS AND ANALYSIS \\ Trial design}

This PATHway trial is a 12-month multicentre, randomised, controlled, assessor and statistician-blinded, parallel, superiority trial with two arms at 1:1 allocation ratio. The study recruitment started in November 2019 and will end in July 2021, with long-term follow-up completed by July 2022. The interventions will take place from baseline to 6 months, and follow-up assessments will occur at 3,6 and 12 months (figure 1). The Consolidated Standards of Reporting Trials statements for non-pharmaceutical trials will be used to guide trial conduct and will be reported according to the Standard Protocol Items: Recommendations for Interventional Trials 2013 Statement. ${ }^{18}$

\section{Participants}

Consecutive patients who present to one of the participating hospitals across the state of New South Wales, Australia, will be invited to participate. Preliminary screening of the expected admissions list will be carried out by the site staff. A short introductory video of the study will be presented to the potential participants. All interested participants will be assessed for potential eligibility by the site staff via an online prescreening survey. Anonymous data on the number of patients declining participation, along with stated reasons for declining, will be collected at each site.

\section{Inclusion criteria}

1. Aged 18 years or older.

2. Has undergone TKR or lumbar laminectomy (with or without fusion).

3. Admission for rehabilitation or surgery to one of the participating study sites.

4. Familiarity with the use of the internet and smart devices (ie, have access and know how to use) and own a smartphone.

5. Ability and willingness to complete study procedures for 12 months.

\section{Exclusion criteria}

1. Known or suspected serious spinal pathology (fracture, metastatic, inflammatory or infective diseases, widespread neurological disorder).

2. Comorbid health conditions that would prevent active participation in the physical activity programmes (eg, unstable angina, uncontrolled hypertension).

3. Inadequate English (reading and speaking) to provide fully informed consent, participate in health coaching sessions or complete outcome measures.

4. Bilateral knee replacement. 


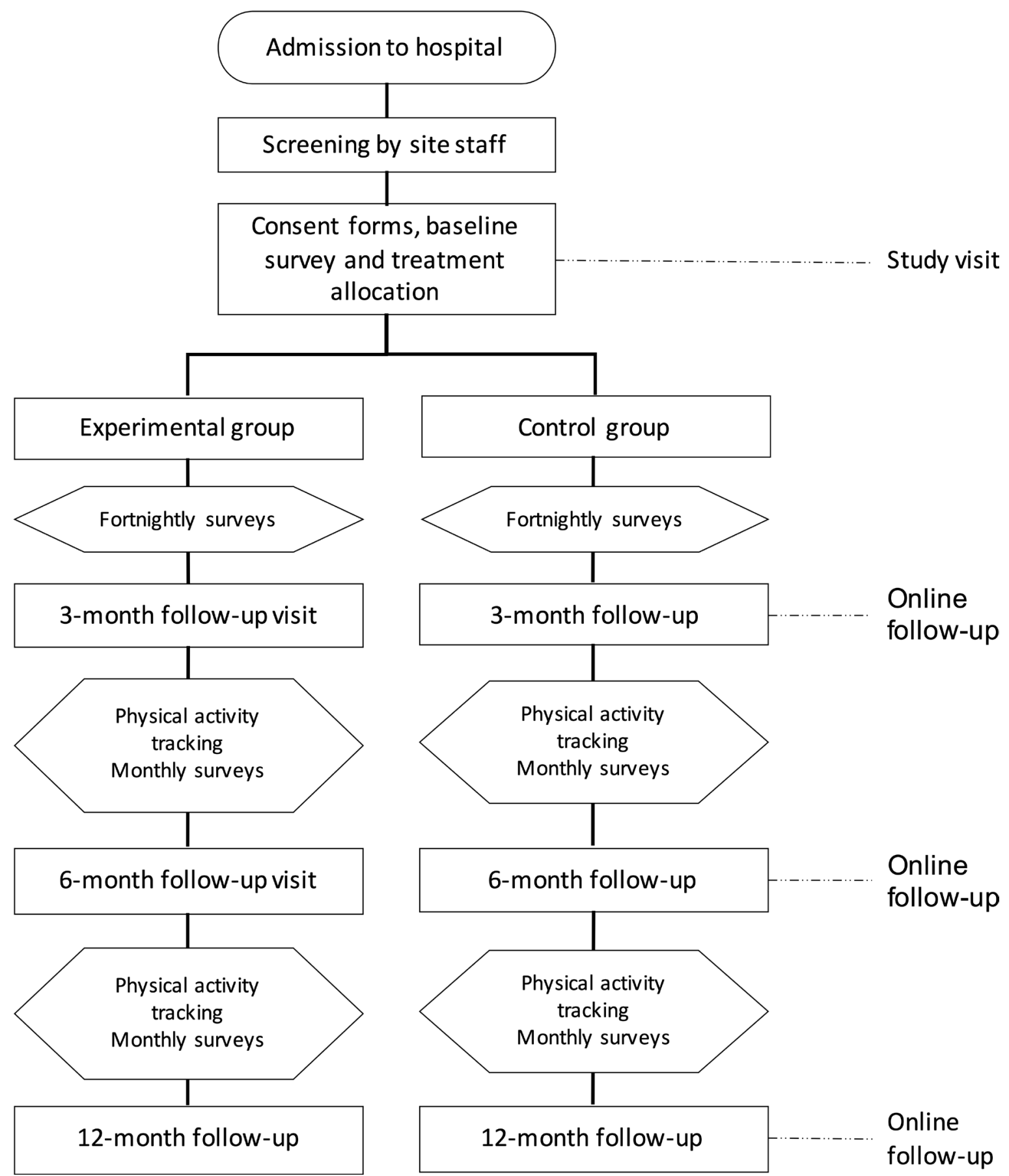

Figure 1 Flow chart of the study design and study procedures.

5. Revision for a previous TKR surgery or laminectomy.

6. Taking part in any other interventional clinical trial.

7. Current use of any intervention component (activity tracker, exercise app or health coaching) and unwillingness to stop using it for the duration of the study.

\section{Randomisation and allocation concealment}

Individuals who qualify and consent to take part in the study will be assigned to either experimental or control group with a 1:1 allocation rate as per computer-generated randomisation schedule using random permuted block sizes of either 2, 4 or 6 and stratified by study sites and surgery type.

The allocation will be concealed by automated assignment using the Research Electronic Data Capture (REDCap) randomisation module. The sequence generation schedules will be prepared by a statistician not involved in the study, and the REDCap randomisation module will be set-up by an independent researcher.

\section{Blinding}

Due to the type of intervention, participants and staff delivering the interventions will not be blinded to the 
group allocation. The site staff, assessors, investigators and study statistician will remain blinded to the treatment allocation until the main results are analysed. Treatment group allocation will be done in REDCap following consent and baseline assessment. To reduce the potential for unblinding, participants will be instructed not to disclose any information about the treatment. In case the assessor is accidentally unblinded, the reason and details of the unblinding process will be recorded.

\section{Interventions}

Usual care: received by both groups

Participants in both groups will receive usual care which is comprised of a standardised clinical pathway (inpatient and outpatient rehabilitation). To ensure that all participants will receive similar major components of the standardised clinical pathway, a PATHway Trial Process Checklist (online supplemental appendix I) was developed in collaboration with the hospital sites for the rehabilitation of patients that have undergone TKR and lumbar laminectomy. The checklist will serve as a guide for the healthcare professionals responsible for the participants' care while an inpatient at the hospital.

\section{Experimental intervention}

The proposed intervention will consist of the clinical pathway augmented by a 6 -month disruptive technology package to optimise engagement and adherence to the postsurgical rehabilitation programme (figure 2). The duration of the intervention is commonly used in previous clinical trials that investigate interventions related to health coaching ${ }^{19}$ or physical activity. ${ }^{20}$ The intervention will be delivered through several apps installed on a study iPad. The technology package will be composed of:

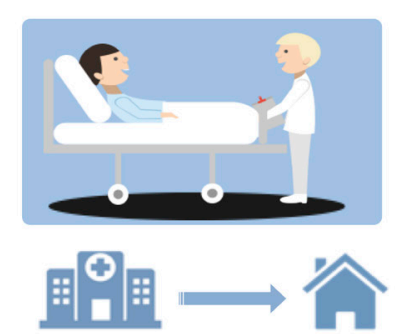

Incorporate wearable technology \& selfmanagement software to optimise patient engagement

Figure 2 Infographic of the intervention components and procedures. 


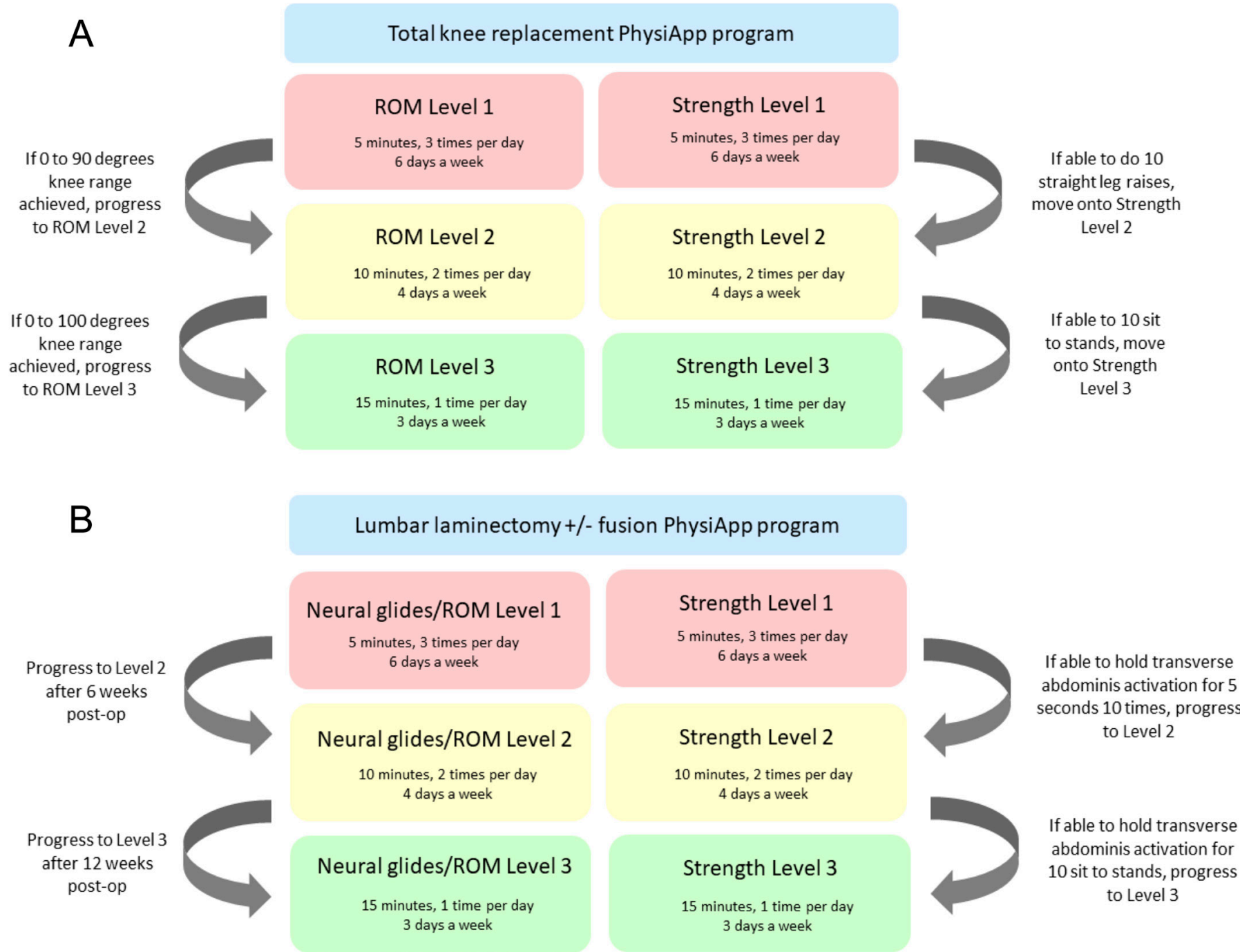

Figure 3 Total knee replacement (A) and lumbar laminectomy (B) exercise programmes.

1. An exercise programme delivered via a health fitness app (PhysiApp) which will be monitored using Physitrack (the clinician portal).

2. A wearable physical activity tracker (Fitbit) built-in with motivational reminders and used in combination with the Fitbit app.

3. Health-coaching delivered via video calls using the Zoom app and via motivational text messages using iMessage app.

4. A knee joint ROM measuring app (Goniometer Pro) for TKR participants.

\section{Exercise program}

A 6-month exercise programme was created for TKR and lumbar laminectomy (figure 3) using Physitrack (Physitrack, UK) by a physiotherapist (VD). The exercise programme focused mainly on strengthening, ROM and neural gliding exercises (the latter was limited to lumbar laminectomy participants). Each programme is comprised of three levels of difficulty with independent progression for strengthening and ROM/neural glide exercises. The exercise programme will be delivered to the participant via PhysiApp, which will record the participant's adherence to the exercises (number sets and repetitions performed). Participants will be asked to rate their pain intensity (Numeric Rating Scale (NRS), 0-10) experienced during exercises.

\section{Activity tracking and progress monitoring}

A commercially available wearable tracker (Fitbit Inspire, Fitbit, USA) will be used to monitor steps, sedentary time, active hours and sleep duration. Participants will be required to use it on a 24/7 basis for 6 months; the use is optional after that. Predefined daily goals were set-up in the device by three stages, and a flagging system will be used to trigger weekly reminders if the goal is not reached (table 1). If the goal is reached, participants will receive a notification/celebratory message.

To monitor participant's progress, adherence and pain intensity during the intervention, a dashboard has been developed using a data analytics platform (Qlik Sense, Qlik Technologies, USA) to display real-time data collected by multiple Fitbit devices and flag if a participant's daily goal is not reached. The dashboard will also 


\begin{tabular}{l} 
Table 1 Qlik Sense flagging system \\
\hline Categories Flags \\
\hline $\begin{array}{l}\text { Step counts } \\
\text { (steps/day) }\end{array}$ \\
$\begin{array}{l}\text { Sedentary } \\
\text { time (hours/ } \\
\text { day) }\end{array}$ \\
$\begin{array}{l}\text { Weekly average }<80 \% \text { of the daily goal } \\
\text { Sleeping } \\
\text { duration } \\
\text { (hours/day) }\end{array}$ \\
Weekly average $<80 \%$ of the daily goal \\
Weekly average $<80 \%$ of the daily goal
\end{tabular}

assist the study coordinators in structuring the health coaching sessions (see the Health-coaching section).

\section{Knee joint ROM}

We will use the Goniometer Pro app for TKR participants, which allows participants to self-assess their active knee ROM which is a crucial indicator of recovery post-TKR. Participants will report their ROM at each health coaching session for 6 months.

\section{Health-coaching}

The 6-month health coaching, alternating video call sessions and motivational text messages, will be delivered by the study coordinators who have been formally trained as qualified health coaches. The video calls will occur fortnightly for 3 months using the Zoom app (Zoom Video Communications, USA), and each will last $30-45 \mathrm{~min}$. The sessions will include (1) monitoring of personal goals for a healthy lifestyle, (2) monitoring of adverse events (AEs), (3) review and progression of exercises, (4) monitoring of knee ROM, (5) tailored motivational advice based on the participant information (exercise adherence, step count, sedentary time and sleep hours) displayed on Physitrack and Qlik Sense. A structured form will be used for guidance. In addition, participants will be provided with motivational text messages delivered via the study iPad regarding exercise adherence, step count, sleeping duration, sedentary time, active hours, pain during exercises and a reminder to sync Fitbit. The messages will be sent fortnightly for the first 3 months and weekly for the following 3 months (after ceasing the video calls).

\section{Outcome measures}

Table 2 provides an overview of measurements according to the Standard Protocol Items: Recommendations for Interventional Trials.

\section{Primary outcome}

Patient-reported average pain intensity assessed on an NRS from 0 (no pain) to 10 (worst pain) over the last week at baseline and 3 months. ${ }^{21}{ }^{22}$ This endpoint was chosen as it is expected that the most pain improvement post surgery will be observed at 3 months. ${ }^{23} 24$

\section{Secondary outcomes}

- Patient-reported average pain intensity assessed on NRS (0-10) over the last week at baseline, 6 and 12 months.

- Patient-reported disability assessed by the Pain Disability Index ${ }^{25}$ at baseline, 3, 6 and 12 months.

- Patient-reported participation in physical activity assessed by the Active Australia Survey ${ }^{26}$ including the number of sessions of physical activity, total time spent in each activity, the proportion of people who were doing a sufficient amount of activity and who were sedentary at baseline, 3, 6 and 12 months.

- Patient-reported sedentary behaviour assessed by the Sedentary Behaviour Questionnaire ${ }^{27}$ at baseline, 3, 6 and 12 months.

- Patient-reported health-related quality of life assessed by the Assessment of Quality of Life-eight items ${ }^{28}$ (AQoL-8) at baseline, 3, 6 and 12 months.

- Raw score and the patient activation levels assessed by the Patient Activation Measure-13 items ${ }^{29}$ (PAM13) at baseline, 3, 6 and 12 months. Patient activation levels were defined as four stages of activation in a progressing difficulty of the items: level 1 -patients believe that their role is important (items 1 and 2); level 2-patients have confidence and knowledge to take action (items 3-8); level 3-taking action (items 9-11) and level 4-staying on course under stress (items 12 and 13). ${ }^{29}$

- Computer self-efficacy assessed by the Modified Computer Self-Efficacy Scale $^{30}$ at baseline and 3 months.

- Objectively measured participation in physical activity using ActivPAL (a physical activity tracking monitor) and Fitbit at 3, 6 and 12 months.

- ActivPAL 5-day measures including

- Sitting/lying time ( $\mathrm{min} /$ day).

- Standing time ( $\mathrm{min} /$ day).

- Stepping time (min/day).

- Number of transitions from sitting to standing.

- Fitbit 5-day measures including

- Step counts (steps/day).

- Sedentary time (hours/day).

- Sleeping duration (hours/day).

- The patient-reported impression of change in pain intensity assessed by the Global Rating of Change ${ }^{31}$ using a 5-point Likert scale at 3,6 and 12 months.

- Healthcare and medication use captured using selfreported questions and data from the Medicare Benefits Scheme (MBS) and Pharmaceutical Benefits Scheme (PBS).

- The 28-day readmission to hospital assessed by a patient-reported question and by MBS/PBS report.

\section{Sample size calculation}

The primary outcome will be average pain intensity (0-10 NRS) in the last week, measured at the 3-month follow-up. A total of 102 participants undergoing TKR will provide $80 \%$ power at a significance level of $5 \%$ to detect 
Table 2 Schedule of study events

\begin{tabular}{|c|c|c|c|c|}
\hline \multirow[b]{2}{*}{ Timepoint } & Study visit & Online follow-up & Online follow-up & Online follow-up \\
\hline & $-T_{1}$ Enrolment & $\begin{array}{l}T_{1} \text { 3-month } \\
\text { follow-up }\end{array}$ & $\begin{array}{l}T_{2} \text { 6-month } \\
\text { follow-up }\end{array}$ & $\begin{array}{l}T_{3} \text { 12-month } \\
\text { follow-up }\end{array}$ \\
\hline \multicolumn{5}{|l|}{ Enrolment } \\
\hline Eligibility screening survey & $x$ & & & \\
\hline Consent checklist and informed consents & $x$ & & & \\
\hline Allocation & $\mathrm{x}$ & & & \\
\hline \multicolumn{5}{|l|}{ Interventions } \\
\hline (Experimental group) & $x$ & $x$ & $x$ & \\
\hline (Control group) & $x$ & $x$ & $x$ & \\
\hline \multicolumn{5}{|l|}{ Assessments } \\
\hline Demographics & $x$ & & & \\
\hline Clinical characteristics & $x$ & & & \\
\hline Comorbidity assessment & $\mathrm{x}$ & & & \\
\hline Pain intensity* & $\mathrm{x}$ & $x$ & $\mathrm{x}$ & $\mathrm{x}$ \\
\hline Pain Disability Index & $x$ & $x$ & $x$ & $x$ \\
\hline Sedentary Behaviour Questionnaire & $x$ & $x$ & $x$ & $x$ \\
\hline AQoL-8D & $x$ & $x$ & $\mathrm{x}$ & $\mathrm{x}$ \\
\hline Patient Activation Measure & $x$ & $x$ & $x$ & $\mathrm{x}$ \\
\hline Modified Computer Self-Efficacy Scale & $x$ & $x$ & & \\
\hline Active Australia Survey & $x$ & $x$ & $x$ & $\mathrm{x}$ \\
\hline 5-day activity tracking & & $x$ & $\mathrm{x}$ & $\mathrm{x}$ \\
\hline $\begin{array}{l}\text { Fortnightly surveys: usage of medical } \\
\text { service/medication }\end{array}$ & & $x$ & & \\
\hline $\begin{array}{l}\text { Monthly surveys: usage of medical service/ } \\
\text { medication }\end{array}$ & & & $\mathrm{x}$ & $x$ \\
\hline Adverse events & & $\mathrm{x}$ & $\mathrm{x}$ & $\mathrm{x}$ \\
\hline Global Rating of Change in Pain & & $\mathrm{X}$ & $\mathrm{x}$ & $\mathrm{X}$ \\
\hline
\end{tabular}

*Numeric Rating Scale 0-10.

AQoL-8D, Assessment of Quality of Life-8 Dimensions with 35 items.

an effect size of 0.6 SDs (ie, a difference between groups of 0.9 point on a 10-point pain NRS), given a pooled SD of 1.5 points $^{32}$; and a drop-out rate of $10 \%$. Likewise, 102 participants undergoing lumbar surgical decompression will provide $80 \%$ power at a significance level of $5 \%$ to detect an effect size of $0.6 \mathrm{SD}$, given an SD of $2.1^{33}$ for this patient subgroup; with a drop-out rate of $10 \%$. A sample size of 102 participants will also provide $70 \%$ power at a significance level of 5\% to detect a difference in AQoL-8D score of 0.1, given a pooled SD of 0.19.

\section{Statistical analysis}

The analysis will be based on the intention-to-treat principle and conducted by surgical procedure (ie, TKR and lumbar laminectomy). Statistical significance will be defined as $\mathrm{p}<0.05$ on the basis of a two-sided test.

Descriptive statistics of baseline characteristics will be summarised by randomisation group and type of conditions (ie, post-TKR and post spinal surgery). Continuous variables will be summarised as mean (SD) where appropriate or median (range). Categorical variables will be summarised as frequency (\%). Pain scores as 3 months will be summarised as mean (SD) or median (range) if non-normal. The primary analysis for the difference in pain will be assessed using t-test if normally distributed or a Mann-Whitney test if nonnormal. Secondary outcomes including a secondary analysis of pain (at 6 and 12 months) will be investigated using generalised estimating equation, this takes into account the repeated measures of the data. Analyses will be performed separately for each condition. A time-by-treatment interaction will be included in the models alongside the main effects to assess the effect of treatment over time. Additional analyses will also be performed, adjusting the models for relevant baseline scores, demographics and clinical characteristics. In case of non-compliance with treatments before the 6-month and 12-month follow-up, a sensitivity analysis (among only those who completed the follow-up questionnaire) will also be performed excluding those who were noncompliant. Missing data will be checked for randomness 
and data missing at random will be imputed using multiple imputations.

A formal interim analysis will be conducted by the independent Data and Safety Monitoring Board (DSMB) after approximately $50 \%$ of participants have completed the primary outcome measurements at 3-month follow-up (primary endpoint). An independent statistician will perform the interim analysis. The DSMB will recommend that the trial continue, continue with modifications to the protocol, or terminate within 2 weeks of completion of a formal interim analysis. A recommendation to terminate early for efficacy should be made only if the DSMB determines there is proof beyond a reasonable doubt that the intervention is effective and outweighs its risks. The Haybittle-Peto stopping rule will be used as a statistical criterion for termination, a difference between groups of 3 SEs favouring the experimental group is clear evidence of a treatment effect. A recommendation to terminate early could also be made based on safety when the DSMB determines (by means they consider appropriate as specified in the DSMB charter) there is proof beyond a reasonable doubt that the experimental intervention causes unacceptable net harm. The trial will not be terminated on the grounds of futility.

A cost-effectiveness analysis will entail a healthcare system perspective. The measure of effectiveness will be quality-adjusted-life-years (QALY) based on measures obtained from the AQoL and transformed into a utility index using weights derived from the Australian population. We will assume no differences in survival between the two arms of the study. Costs to the healthcare system will be based on MBS and PBS costs. Costs of hospitalisations will be valued at standard Australian refined diagnosisrelated groups (AR-DRG) cost weights. Costs of the study treatments and private healthcare services will be valued at published standard rates, if available, or as reported by participants in their diaries. The aggregate of such costs will be used to estimate healthcare costs incurred by patients in both arms of the trial. Difference in QALYs will be calculated as the difference in the area under the curve for AQoL-8D scores at 3, 6 and 12 months. Differences in mean AQoL-8D change from baseline will be weighted by time from baseline using generalised linear regression models adjusted for baseline AQoL-8D to construct QALYs, and compared between groups. Differences in mean cost between groups will be calculated using generalised linear regression models. Incremental cost per QALY will be calculated as the ratio of difference in mean cost to difference in mean QALYs between treatment arms. Sensitivity analyses on the most important cost drivers will be performed to assess the robustness of the results, and a $95 \%$ CI for net benefits will be calculated based on a range of values for the social willingness to pay for a QALY.

\section{Patient and public involvement}

Patient representatives and clinicians were initially consulted regarding the study materials, study procedures and burden of the intervention and time required to participate in the research. Their feedback was used to produce the final version of the protocol. Furthermore, we will actively involve them in the development of the dissemination approaches and resources and will work collaboratively with them in future implementation.

\section{Participant safety and withdrawal}

The risks for participants involved in this study are minimal. However, to ensure the safety of the participants, we will monitor them for any potential AEs at every follow-up timepoint (3, 6 and 12 months). The expected adverse reactions are delayed-onset muscle soreness and a temporary increase in joint pain due to exercises and augmentation in physical activity levels. Participants reporting high pain intensity ( $\geq 8$ out of 10 ) in one or more exercises on PhysiApp will have a video call arranged with the study coordinator and the study physiotherapist. Exercise performance will be assessed and corrected. If necessary, the exercise will be replaced, or the programme will be modified. Additionally, participants might experience skin reactions from wearing the ActivPAL or Fitbit. All AEs occurring after the entry into the study will be recorded and documented in REDCap indicating the start and end date of the event, details of the event, any actions taken and the outcome. The DSMB will be notified of all AEs to ensure the safety of participants.

A participant can leave the research study at any time. When withdrawing from the study, the participant should inform the research team that he/she wishes to withdraw by signing and returning a withdrawal form. The participant may provide the research team with the reason(s) for leaving the study but is not required to do so. Strategies (eg, survey reminders, emails, phone calls and text messages) will be used to maximise follow-up and prevent missing data, including adhering to the assessment schedule in the event of participant withdrawal. Participants included in the study will also be allowed to keep the devices received after the end of the study. Participants who withdraw from the study will not be replaced.

\section{Data security and handling}

Study data will be collected and managed using REDCap tool hosted at the University of Sydney. This tool is a secure, web-based application designed to support data capture for research studies, providing: (1) an intuitive interface for validated data entry; (2) audit trails for tracking data manipulation and export procedures; (3) automated export procedures for seamless data downloads to common statistical packages and (4) procedures for importing data from external sources. Back-up of re-identifiable information will be kept in passwordprotected electronic files. The privacy, security and ownership of the research data will be maintained and will not be stored or accessible by another organisation. Physical documents containing identifiable information will be stored in a locked cabinet throughout the 
study. Any files containing identifiable information will be encrypted before being transmitted via CloudStor FileSender.

In compliance with the NSW State Records Act, the archiving period for clinical research records will be 15 years. ${ }^{34}$ After this period, the electronic files will be deleted, and paper forms will be destroyed. No information which could lead to the identification of a participant will be included in the dissemination of results. All investigators will have full access to the complete final dataset. A de-identifiable dataset containing individual participant data will be published in an open-access Data Repository 3 years after study close-out for sharing purposes.

\section{Ethics and dissemination}

This protocol, study-related documents and any subsequent modifications will be reviewed and approved by the sponsor (the University of Sydney) and research ethics committee. Any protocol modification will be amended at the trial registry. We will disseminate the results of this research in lay summary, infographics, conferences, reports, scientific publications and $\mathrm{PhD}$ thesis which will all be in an audience-specific manner. Potential participants must provide written informed consent to the recruiting physiotherapist before entering the study. We will actively involve and collaborate with stakeholders/ knowledge users (consumers, clinicians and so on) in the production and implementation of these resources.

\section{Author affiliations}

${ }^{1}$ Institute of Bone and Joint Research, The University of Sydney, Sydney, New South Wales, Australia

${ }^{2}$ Department of Rheumatology, The University of Sydney, Sydney, New South Wales, Australia

${ }^{3}$ Research Assets Division, Sax Institute, Sydney, New South Wales, Australia ${ }^{4}$ Centre for Health Economics, Monash University, Melbourne, Victoria, Australia ${ }^{5}$ University of Sydney Institute of Bone and Joint Research, Saint Leonards, New South Wales, Australia

\section{Twitter David J Hunter @ProfDavidHunter}

Acknowledgements We acknowledge all study participants, trial staff, all recruiting hospitals and site staff, especially the physiotherapists and allied health managers.

Contributors DJH and MF attained project funding. DJH, MF, LM, SR, SC, XW and VD contributed to study conception and design. AH contributed to the design of the economic evaluation and will perform the cost-effectiveness analysis. DJH, MF, LM, SR, SC, XW, VD and AH revised the protocol critically for important intellectual content and read and approved the final version of the protocol. DJH, MF, LM, SR, $\mathrm{SC}, \mathrm{XW}, \mathrm{VD}$ and $\mathrm{AH}$ agree to be accountable for all aspects of the work.

Funding This work has been supported by the Ramsay Hospital Research Foundation. The company, MKM Health Pty Ltd, developed a data extract routine to export the activity data from the participant's Fitbit account and set up the Qlik Sense dashboard to analyse, investigate and visualise the activity data. DJH is supported by an NHMRC Practitioner Fellowship. MF is funded by a National Health and Medical Research Council (NHMRC) Career Development Fellowship.

Disclaimer The funder and other sources listed above had no role in the design of this study and will have no role during its execution, analysis, data interpretation, manuscript writing or dissemination of results.

Competing interests DJH is a consultant to Pfizer, Lilly, TLCBio and Merck Serono. Patient consent for publication Not required.

Provenance and peer review Not commissioned; externally peer reviewed.
Supplemental material This content has been supplied by the author(s). It has not been vetted by BMJ Publishing Group Limited (BMJ) and may not have been peer-reviewed. Any opinions or recommendations discussed are solely those of the author(s) and are not endorsed by BMJ. BMJ disclaims all liability and responsibility arising from any reliance placed on the content. Where the content includes any translated material, BMJ does not warrant the accuracy and reliability of the translations (including but not limited to local regulations, clinical guidelines, terminology, drug names and drug dosages), and is not responsible for any error and/or omissions arising from translation and adaptation or otherwise.

Open access This is an open access article distributed in accordance with the Creative Commons Attribution Non Commercial (CC BY-NC 4.0) license, which permits others to distribute, remix, adapt, build upon this work non-commercially, and license their derivative works on different terms, provided the original work is properly cited, appropriate credit is given, any changes made indicated, and the use is non-commercial. See: http://creativecommons.org/licenses/by-nc/4.0/.

ORCID iDs

Xia Wang http://orcid.org/0000-0003-3172-9390

David J Hunter http://orcid.org/0000-0003-3197-752X

\section{REFERENCES}

1 GBD 2017 Disease and Injury Incidence and Prevalence Collaborators. Global, regional, and national incidence, prevalence, and years lived with disability for 354 diseases and injuries for 195 countries and territories, 1990-2017: a systematic analysis for the global burden of disease study 2017. Lancet 2018;392:1789-858.

2 AlHW. Australian Institute of health and welfare 2019. musculoskeletal conditions and comorbidity in Australia. arthritis series No. 25. cat. No. Phe 241. Canberra: AlHW, 2019.

3 Australia M. Medicare statistics Canberra Australian government, 2020. Available: http://medicarestatistics.humanservices.gov.au/ statistics/mbs item.jsp

4 Australian Institute of Health and Welfare. Health expenditure Australia 2013-14. contract NO: cat. No. HWE 63. Canberra: AlHW, 2015.

5 Liddle AD, Judge A, Pandit $\mathrm{H}$, et al. Adverse outcomes after total and unicompartmental knee replacement in 101,330 matched patients: a study of data from the National joint Registry for England and Wales. Lancet 2014;384:1437-45.

6 Naylor J, Harmer A, Fransen M, et al. Status of physiotherapy rehabilitation after total knee replacement in Australia. Physiother Res Int 2006;11:35-47.

7 Buhagiar MA, Naylor JM, Harris IA, et al. Effect of inpatient rehabilitation vs a monitored home-based program on mobility in patients with total knee arthroplasty: the $\mathrm{HIHO}$ randomized clinical trial. JAMA 2017;317:1037-46.

8 Campbell R, Evans M, Tucker M, et al. Why don't patients do their exercises? Understanding non-compliance with physiotherapy in patients with osteoarthritis of the knee. J Epidemiol Community Health 2001;55:132-8.

9 Levinger P, Zeina D, Teshome AK, et al. A real time biofeedback using Kinect and Wii to improve gait for post-total knee replacement rehabilitation: a case study report. Disabil Rehabil Assist Technol 2016;11:251-62.

10 Fung V, Ho A, Shaffer J, et al. Use of Nintendo Wii Fit ${ }^{T M}$ in the rehabilitation of outpatients following total knee replacement: a preliminary randomised controlled trial. Physiotherapy 2012;98:183-8.

11 Kwasnicki RM, Ali R, Jordan SJ, et al. A wearable mobility assessment device for total knee replacement: a longitudinal feasibility study. Int J Surg 2015;18:14-20.

12 Skrepnik N, Spitzer A, Altman R, et al. Assessing the impact of a novel smartphone application compared with standard follow-up on mobility of patients with knee osteoarthritis following treatment with Hylan G-F 20: a randomized controlled trial. JMIR Mhealth Uhealth 2017;5:e64.

13 Krein SL, Kadri R, Hughes M, et al. Pedometer-based internetmediated intervention for adults with chronic low back pain: randomized controlled trial. J Med Internet Res 2013;15:e181.

14 Nussbaum R, Kelly C, Quinby E. A systematic review of mobile health applications in rehabilitation. Arch Phys Med Rehabil 2018;29:31175-4.

15 Schoeppe S, Alley S, Van Lippevelde W, et al. Efficacy of interventions that use apps to improve diet, physical activity and sedentary behaviour: a systematic review. Int J Behav Nutr Phys Act 2016;13:127. 
16 Gal R, May AM, van Overmeeren EJ, et al. The effect of physical activity interventions comprising Wearables and smartphone applications on physical activity: a systematic review and metaanalysis. Sports Med Open 2018:4:42.

17 Wang X, Hunter DJ, Vesentini G, et al. Technology-Assisted rehabilitation following total knee or hip replacement for people with osteoarthritis: a systematic review and meta-analysis. BMC Musculoskelet Disord 2019;20:506.

18 Chan A-W, Tetzlaff JM, Altman DG, et al. Spirit 2013 statement: defining standard protocol items for clinical trials. Ann Intern Med 2013;158:200-7.

19 Dejonghe LAL, Becker J, Froboese I, et al. Long-Term effectiveness of health coaching in rehabilitation and prevention: a systematic review. Patient Educ Couns 2017;100:1643-53.

20 Sansano-Nadal O, Giné-Garriga M, Brach JS, et al. Exercise-Based interventions to enhance long-term sustainability of physical activity in older adults: a systematic review and meta-analysis of randomized clinical trials. Int J Environ Res Public Health 2019;16. doi:10.3390/ ijerph16142527. [Epub ahead of print: 1507 2019].

21 Pengel LHM, Refshauge KM, Maher CG. Responsiveness of pain, disability, and physical impairment outcomes in patients with low back pain. Spine 2004;29:879-83.

22 Scrimshaw SV, Maher C. Responsiveness of visual analogue and McGill pain scale measures. J Manipulative Physiol Ther 2001;24:501-4.

23 Farrar JT, Young JP, LaMoreaux L, et al. Clinical importance of changes in chronic pain intensity measured on an 11-point numerical pain rating scale. Pain 2001;94:149-58.

24 Lenguerrand E, Artz N, Marques E, et al. Effect of group-based outpatient physical therapy on function after total knee replacement: results from a multicenter randomized controlled trial. Arthritis Care Res 2020;72:768-77.
25 Tait RC, Chibnall JT, Krause S. The pain disability index: psychometric properties. Pain 1990;40:171-82.

26 Brown WJ, Trost SG, Bauman A, et al. Test-Retest reliability of four physical activity measures used in population surveys. J Sci Med Sport 2004;7:205-15.

27 Marshall AL, Miller YD, Burton NW, et al. Measuring total and domain-specific sitting: a study of reliability and validity. Med Sci Sports Exerc 2010;42:1094-102.

28 Hawthorne G. Assessing utility where short measures are required: development of the short assessment of quality of Life-8 (AQoL-8) instrument. Value Health 2009;12:948-57.

29 Hibbard JH, Mahoney ER, Stockard J, et al. Development and testing of a short form of the patient activation measure. Health Serv Res 2005;40:1918-30.

30 Laver K, George S, Ratcliffe J, et al. Measuring technology self efficacy: reliability and construct validity of a modified computer self efficacy scale in a clinical rehabilitation setting. Disabil Rehabil 2012;34:220-7.

31 Kamper SJ, Maher CG, Mackay G. Global rating of change scales: a review of strengths and weaknesses and considerations for design. $J$ Man Manip Ther 2009;17:163-70.

32 Wang X, Ferreira M, Hunter D, et al. Digital Disruptive Technology for Rehabilitation Following Elective Surgery for Low Back Pain, Knee and Hip Osteoarthritis: A Systematic Review and Meta-Analysis [abstract].. Arthritis Rheumatol 2018;70:suppl 10.

33 Mannion AF, Denzler R, Dvorak J, et al. A randomised controlled trial of post-operative rehabilitation after surgical decompression of the lumbar spine. Eur Spine J 2007;16:1101-17.

34 New South Wales supplement to the National statement, section 3.3.112011. 